OPEN ACCESS

Edited by:

Sheng Li.

The University of Texas Health Science Center at Houston, USA

Reviewed by:

Fan Gao,

University of Texas Southwestern Medical Center, USA

Kelly J. Cole,

University of lowa, USA

*Correspondence:

Wei Zhang

wei.zhang@csi.cuny.edu

Received: 03 October 2016 Accepted: 09 November 2016

Published: 25 November 2016

Citation:

Carteron A, McPartlan K, Gioeli C

Reid E, Turturro M, Hahn B,

Benson C and Zhang W (2016) Temporary Nerve Block at Selected Digits Revealed Hand Motor Deficits in Grasping Tasks.

Front. Hum. Neurosci. 10:596. doi: 10.3389/fnhum.2016.00596

\section{Temporary Nerve Block at Selected Digits Revealed Hand Motor Deficits in Grasping Tasks}

\author{
Aude Carteron ${ }^{1}$, Kerry McPartlan ${ }^{1}$, Christina Gioeli ${ }^{1}$, Emily Reid ${ }^{1}$, Matt Turturro ${ }^{1}$, \\ Barry Hahn ${ }^{2}$, Cynthia Benson ${ }^{2}$ and Wei Zhang ${ }^{1,3 *}$ \\ ${ }^{1}$ Department of Physical Therapy, College of Staten Island, City University of New York, Staten Island, NY, USA, ${ }^{2}$ Emergency \\ Medicine, Staten Island University Hospital, Staten Island, NY, USA, ${ }^{3}$ Ph.D. Program in Biology, Graduate School and \\ University Center, City University of New York, New York, NY, USA
}

Peripheral sensory feedback plays a crucial role in ensuring correct motor execution throughout hand grasp control. Previous studies utilized local anesthesia to deprive somatosensory feedback in the digits or hand, observations included sensorimotor deficits at both corticospinal and peripheral levels. However, the questions of how the disturbed and intact sensory input integrate and interact with each other to assist the motor program execution, and whether the motor coordination based on motor output variability between affected and non-affected elements (e.g., digits) becomes interfered by the local sensory deficiency, have not been answered. The current study aims to investigate the effect of peripheral deafferentation through digital nerve blocks at selective digits on motor performance and motor coordination in grasp control. Our results suggested that the absence of somatosensory information induced motor deficits in hand grasp control, as evidenced by reduced maximal force production ability in both local and non-local digits, impairment of force and moment control during object lift and hold, and attenuated motor synergies in stabilizing task performance variables, namely the tangential force and moment of force. These findings implied that individual sensory input is shared across all the digits and the disturbed signal from local sensory channel(s) has a more comprehensive impact on the process of the motor output execution in the sensorimotor integration process. Additionally, a feedback control mechanism with a sensation-based component resides in the formation process for the motor covariation structure.

Keywords: hand, anesthesia, anticipatory control, force, moment, motor coordination, motor synergy

\section{INTRODUCTION}

Peripheral sensory feedback plays a crucial role in the correct execution of a voluntary movement (Nowak et al., 2003, 2004; Weiss et al., 2011; Stone and Gonzalez, 2015). A continuously updated cyclic sensorimotor integration process is required to successfully complete a motor task. Each cycle begins with peripheral pathways conveying sensory information, followed with the central nervous system integrating the sensory inputs to assist motor program execution (Abbruzzese and Berardelli, 2003). Thus, abnormalities in the peripheral afferent inputs, or in their central processing, may interfere with motor program execution, leading to motor function deficits (Rossini et al., 1996; Rossi et al., 1998; Nowak et al., 2003; Richardson et al., 2016). Additionally, 
those abnormalities partially contribute to the motor disorder under pathophysiological conditions (Hallett, 1995; Kaji et al., 1995; Fellows et al., 1998; Marchese et al., 2000; Quinn et al., 2001; Schwarz et al., 2001; Sens et al., 2013; Zhang and Santello, 2014). Despite the apparent importance of afferent inputs in the process of sensorimotor integration, its explicit role in voluntary movement control mechanism is not well understood.

To explore the contribution of somatosensory feedback in hand manipulation tasks, object grasping has been used as an effective model (Lederman and Klatzky, 1990, 2009; Stone and Gonzalez, 2014a,b). Previous studies revealed that absent or disturbed afferent inputs interfered with the sensorimotor integration process during steady or dynamic prehensile tasks. Local anesthesia procedures have been applied to individual digits (Witney et al., 2004) or to the lower median nerve (Li et al., 2004; Li and Nimbarte, 2006) to block peripheral somatosensory feedback (Gissen et al., 1982; Witney et al., 2004). These studies suggested that digital deafferentation introduced multiple motor deficits in force modulations. Force deficits include deteriorated maximal grip force production (Augurelle et al., 2003; Pavlova et al., 2015), excessively large grip force to lift or hold an object (Johansson and Westling, 1984; Johansson et al., 1992; Nowak et al., 2001; Monzée et al., 2003; Dun et al., 2007), delayed force development or force adaptation to perturbation (Johansson and Westling, 1984; Johansson et al., 1992; Jenmalm and Johansson, 1997; Monzée et al., 2003), disturbed coupling of normal and tangential forces (Nowak et al., 2001; Augurelle et al., 2003), and inaccurate moment of force production patterns (Monzée et al., 2003; Li and Li, 2013). Although none of these studies investigated the whole hand grasp control (i.e., only twodigit pinch or three-digit grasp was employed), their findings revealed an impaired sensorimotor integration process following somatosensory feedback deprivation at peripheral levels. At corticospinal levels, neurophysiological studies suggested that cutaneous feedback deprivation decreases the activation of motor neurons in several parts of the central nervous system (Rossini et al., 1996; Rossi et al., 1998), such as the dorsal horn of the spinal cord (Koerber and Brown, 1995), thalamus (Nicolelis et al., 1993; Rasmusson, 1996), and the cerebral cortex (Wall et al., 1986). However, most previous studies focused on the effect of local afferent abnormalities on adaptive peripheral behavioral and central processing changes, and none of them addressed the following questions: (1) how the disturbed and intact sensory input integrate and interact with each other to modulate the motor program execution; and (2) whether the motor coordination based on motor output variability presents between affected and non-affected elements (e.g., digits) and becomes interfered by the local sensory deficiency.

Some recent studies explored the sensorimotor integration process in carpal tunnel syndrome (CTS) patients (Zhang and Santello, 2014). Given reduced tactile sensation solely at a subset of digits (i.e., the thumb, index, middle and lateral half of the ring finger), CTS patients demonstrated excessive grip force (Zhang et al., 2011), decreased motor learning and adaptation (Zhang et al., 2012), lower force discrimination, and non-efficient torque production (Zhang et al., 2011). Most interestingly, the patients' excessively large force was presented only when the non- or less-affected fingers were employed together with CTS-affected digits in the grip task (Zhang et al., 2013). These findings suggest a larger challenge for the CNS to integrate disturbed with intact peripheral sensory channels in a motor task adaptation. The purpose of the current study is to investigate the multi-digit motor performance and coordination within and between deafferent and intact digits during hand grasping control tasks. Digital nerve block was applied to temporarily remove the somatosensory feedback from a subset of digits, but not the entire hand. We expect that the absence of somatosensory input from the digits would induce motor deficits in both grasp force control and moment control, and would deteriorate the motor coordination between deafferent and intact digits.

\section{MATERIALS AND METHODS}

\section{Subjects}

Nineteen (10 females and 9 males) right-handed young adults participated in the current study (age (mean \pm SD): $23.32 \pm$ 0.37 years old, height: $1.69 \pm 0.02 \mathrm{~m}$, weight: $72.35 \pm 2.99 \mathrm{~kg}$, hand width and length: $8.28 \pm 0.17 \mathrm{~cm}$ and $18.11 \pm 0.25 \mathrm{~cm}$ respectively). Subjects had no revealed history of neurological, musculoskeletal, vascular or metabolic disorders, impairment of their right upper limb, or allergy history for the anesthetic agent and preparation material. The study protocol was approved by the Institutional Review Board at the City University of New York and North Shore-Long Island Jewish Health system. All subjects were naïve to the purpose of the study and gave their written informed consent according to the Declaration of Helsinki.

\section{Apparatus}

An inverted T-shape customized grip device was applied in the current study to allow for comfortable grasp. Five force/torque (F/T) transducers (Nano-25 for the thumb and Nano-17 for four fingers, ATI Industrial Automation, Apex, NC, USA) were installed to measure local forces and torques in three dimensions at individual digits, i.e., thumb, index, middle, ring and little (T, I, M, R and L respectively; Figure 1A). Note that when only three digits were used in the grasp tasks $(3 \mathrm{D}$, see procedure below), the $\mathrm{F} / \mathrm{T}$ sensor for the thumb and the two intermediate sensors on the fingers side were used instead (Figure 1B). All sensors were covered by 100-grit sandpaper to increase grip friction and prevent slipping. One electromagnetic tracking sensor (Polhemus Fastrak, Colchester, VT, USA; $0.075 \mathrm{~mm}$ and $0.05 \mathrm{u}$ resolution) was attached on the top of the device to measure its position/orientation $(\mathrm{P} / \mathrm{O})$. The grip device was loaded with a $200 \mathrm{~g}$ mass in the center at the bottom to centralize its mass distribution and achieve adequate yet comfortable weight (665 $\mathrm{g}$ in total) for the lifting task. Note that both the mass and mass distribution remained the same throughout the experiment. Dimensions of the device are indicated in Figures 1A,B. Five 12-bit A/D converted boards recorded the 


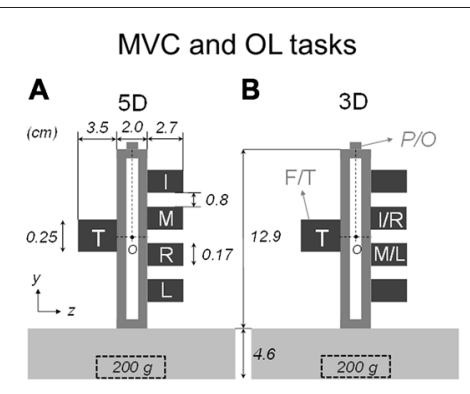

$5 \mathrm{D}$
Martini glass task

C

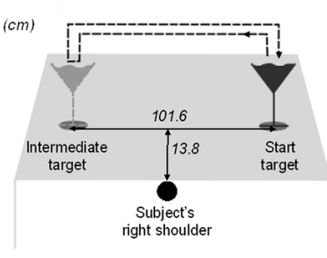

3D
D

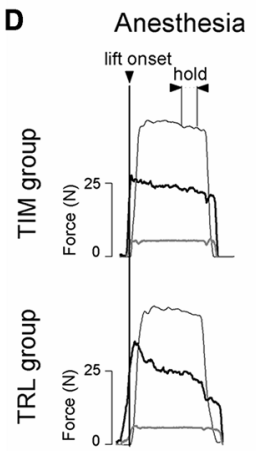

Control ${ }^{2}$
$-F_{G}$

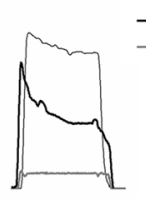

Anesthesia

Control $-F_{T}$
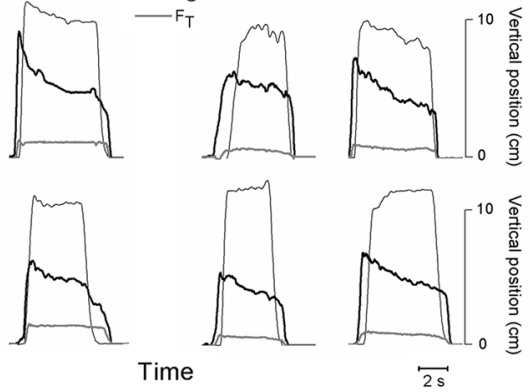

FIGURE 1 | Experimental setup and variables. $(\mathbf{A}, \mathbf{B})$ Show the front view of the customized grip device utilized for maximal voluntary contraction (MVC) and object lifting (OL) tasks. Five force/torque (F/T) sensors are mounted on both sides of the device to measure forces and moment of forces exerted by individual digits involved in five-digit grip (5D; thumb, index, middle, ring and little fingers: T, I, M, R and L, respectively) in (A) or three-digit grip (3D; TIM or TRL) in (B). A mass of $200 \mathrm{~g}$ was inserted in the midpoint at the bottom of the grip device throughout the tasks. Note that all five sensors were mounted on the grip device to maintain a constant weight for both grip types, even though only the two intermediate sensors were used for I and $\mathrm{M}$ (or $\mathrm{R}$ and $\mathrm{L}$ ) in 3D. A magnetic tracker position/orientation $(\mathrm{P} / \mathrm{O})$ attached on the top of the device was used to measure the object position and orientation during the manipulation. "O" denotes the point about which moments were computed (see "Materials and Methods" Section for more details). (C) Demonstrates an auxiliary task (Martini glass task) when a martini glass filled with water was to be lifted by using a five-digit grip, transported from a start target location to an intermediate target location, and transported back to the origin location. A dashed arrow line denotes the glass transportation trajectory. Subject's right shoulder was aligned with the center between the two target locations. (D) Shows the time course of object vertical position $\left(P_{\mathrm{Z}}\right)$, grip force $\left(\mathrm{F}_{\mathrm{G}}\right)$ and tangential force $\left(\mathrm{F}_{\mathrm{T}}\right)$ performed by one representative subject before (Control) and after digital nerve block procedure (Anesthesia) from either TIM or TRL group by using three- and five-digit grip types. All data traces are aligned with object lift onset (vertical line). Plotted data in each subpanel are from a subsequent trial in $\mathrm{OL}$ task. Experimental variables were analyzed and extracted at two defined task epochs: at object lift onset and during object hold.

$\mathrm{F} / \mathrm{T}$ data at a sampling frequency of $1 \mathrm{kHz}$, and $\mathrm{P} / \mathrm{O}$ data were recorded at $80 \mathrm{~Hz}$. All the measurements were acquired, displayed and stored through a customized program in LabVIEW 2010 (National Instruments).

\section{Experimental Procedures}

All subjects completed the current study in two sessions with a 2-week interval: the first session will be referred to as control session while the second one, where a standard digital nerve block procedure was conducted, will be referred to as anesthesia session. Subjects were assigned randomly into one of two groups according to the digits to be anesthetized during the anesthesia session: TIM group (5F, 5M) and TRL group (5F, 4M), with digital anesthesia applied for thumb, index and middle and for thumb, ring and little fingers separately.

In both control and anesthesia sessions, each subject was instructed to complete two primary experimental tasks, including a maximal voluntary contraction (MVC) task and an object lifting (OL) task. Additionally, subjects completed an auxiliary experimental task, i.e., martini glass task. During the experiment, subjects sat comfortably 10-15 cm away from a test table and faced a grip object (a grip device or a martini glass according to the task). The grip object was placed within arm's reach to allow comfortable reaching with minimal trunk movement. The grip device was not fixed on the top of the desk throughout the experiment. Before the experiment, subjects were asked to rest their right hand on the table without touching the grip object. For both MVC and OL tasks, the subject's right shoulder was aligned with the grip device.

In the MVC task, subjects' peak grip force were measured to evaluate their maximal force production ability with the hand in prehensile position. After a verbal "go" signal, subjects were instructed to reach the grip device from a hand rest position, contact the tip of all task-involved digits on corresponding individual $\mathrm{F} / \mathrm{T}$ sensors, squeeze as strong as possible within $8 \mathrm{~s}$ without lifting the device from the top of the desk, and relax after their peak maximal force was reached. Subjects were provided with an online feedback by displaying their total grip force-time profile on a computer monitor. Displayed force was always scaled to subject's maximal force amplitude to ensure visual feedback for full range of force. MVC task consisted of three grip conditions according to the digits combination instructed to be used, namely: (1) 5D: all five digits; (2) 3D_ane: all three digits selected to be anesthetized in the anesthesia session (i.e., T, I and $\mathrm{M}$ for TIM group, and T, R and L for TRL group); and (3) 3D_mix: a three digit combination of the thumb and two non-anesthetized fingers (i.e., T, R and L for TIM group, and T, I and M for TRL group). Subjects completed two trials for each condition resulting in six trials in total in MVC task.

In OL task, subjects were instructed to reach the grip device after a verbal "go" signal, contact task-involved digits on the surface of individual sensors, lift the grip device $\sim 10 \mathrm{~cm}$ above the table at a self-selected pace, hold it in the air for $4 \mathrm{~s}$, and replace it on the table. Two different grip types were used in OL task: a five-digit and three-digit grip (5D and 3D, respectively). Note that the three digits used in the 3D grip were all anesthetized digits (i.e., T, I and M for TIM group, and T, R and L for TRL group). After three practice trials, subjects performed 25 trials with each grip type, resulting in 56 trials in total in OL task. The instruction "lift and hold the object as vertical as possible" was consistently repeated throughout the OL task for all the subjects.

The auxiliary experimental task, martini glass task (Figure 1C), was designed to mimic a daily-life hand activity 
with the addition of interference to the sensorimotor integration process. Before the martini glass task, subjects rested their right hand on the desk, which was aligned with their right shoulder and the midpoint between "Start target" and "Intermediate target", the original and intermediate position of the martini glass respectively (Figure 1C). After a verbal "go" signal, subjects reached their right hand to a standard martini glass filled with water (436 g including $352 \mathrm{~g}$ of water) located at "Start target", gripped it by placing their five digits on the glass stem only, transported it to "Intermediate target" without releasing nor changing digit placement, and replaced it back at "Start target". Subjects were required to complete the martini glass transportation as quick as possible while minimizing water spill. Each subject performed the martini glass task for three successive trials. The time duration of each trial was recorded and the cumulated mass of water spilled over the three trials was calculated after weighting the martini glass with the water remaining.

At least $30 \mathrm{~s}$ between-trials and 5 min between-tasks pauses were observed during the experiment. Subjects were given necessary rest time to prevent fatigue and additional rest time upon individual request.

\section{Digital Nerve Block at Selective Digits}

During the anesthesia session, a standard digital nerve block procedure was conducted by the same licensed physician (Staten Island University Hospital, New York, NY, USA) for all the subjects prior to the experimental tasks described above. Local anesthetic ( $1 \%$ lidocaine and $0.5 \%$ bupivicaine) injections were administered at digital nerves gradually and incrementally in the web space so that sensory nerve fibers were blocked including type C, type A delta, gamma and beta (pain, temperature, postural, touch and pressure), while motor nerve fibers (type A alpha) remained intact (Gissen et al., 1982) ${ }^{1}$. The physician determined the dosage of medication according to individual subject body weight. Additional dosage was injected if residual tactile sensations remained after initial injection, and up to three injections were conducted for the same digit per subject. Participants whose tactile sensation was not successfully blocked after receiving three injections were excluded from the study. A microfilament toolkit was used for all digits after each digital nerve block procedure and throughout the experiment to ensure the blockage of tactile sensation at three anesthetized digits but not at two un-anesthetized digits.

\section{Data Processing}

Data analyses were performed with MATLAB 8.1.0 (MathWorks), Excel 14.0 (Microsoft) and SPSS 20 (IBM). Data from one subject (female in TIM group) was determined as

\footnotetext{
${ }^{1}$ Sensory neuron fibers are generally more sensitive to digital nerve block than motor neuron fibers according to a characteristic of local anesthetic actions: nerves with higher firing frequency and more positive membrane potential are more sensitive to local anesthetic block. Sensory fibers, especially pain fibers, have a high firing rate and relatively longer action potential duration than motor fibers, and thus are more sensitive to lower concentrations of local anesthetics.
}

an outlier, i.e., out of range of mean $\pm 3 \mathrm{SD}$, and was therefore omitted from our data analysis and results. Figure 1D shows time courses of kinetic and kinematic data from representative subjects in TIM and TRL groups before and after their digital nerve block procedure (Control and Anesthesia respectively) by using three- or five-digit grip types.

For convenience, virtual finger (VF), a hypothetical entity that produces a mechanical output equivalent to the individual fingers combined (Arbib et al., 1985; Baud-Bovy and Soechting, 2001; Zhang et al., 2009), is used to denote all task-involved digits except the thumb. In the current study, we defined two substitutes: VFa to designate the set of two fingers that received the injection during the anesthesia visit (i.e., I and $M$ for TIM subject group; R and L for TRL subject group), and VFna to designate the set of two non-anesthetized fingers on the VF side (i.e., R and L for TIM subject group; I and M for TRL subject group). In this scenario, different grip condition could be viewed as a combination of T, VFa and VFna: $5 \mathrm{D}$ involved $\mathrm{T}, \mathrm{VF} a$ and VFna, whereas 3D grip conditions involved T and VF $a$ or VFna.

In OL task, kinematics and kinetics data were temporally aligned offline by re-sampling position and orientation data through linear interpolation to match the force data sampling frequency. Experimental variables were extracted at two defined task epochs: (1) at object lift onset; and (2) during object hold. Object lift onset reflects anticipatory control behavior based on previous trials, whereas object hold provides an insight into motor adaptation resulting from the integration of sensory feedback acquired following lift onset (Zhang et al., 2010, 2011, 2012, 2013). These task epochs were determined for each individual trial based on the smoothed position data. Briefly, object lift onset was identified as the time when the derivative of the object's vertical position $(\mathrm{Pz})$ crossed a threshold (signal baseline mean +3 SD) for more than $200 \mathrm{~ms}$. The end of object hold was determined as the time during object release when the derivative of $\mathrm{Pz}$ dropped below $3 \%$ of its baseline at hold. As force transients occur at the beginning and by the end of the object hold period, experimental variables related to object hold were analyzed by averaging over the $1.2 \mathrm{~s}$ period of the steady portion $0.5 \mathrm{~s}$ prior to the end of object hold (Figure 1D).

All the mechanical variables of interest were in the frontal plane (Figures 1A,B). Experimental kinetics variables in MVC and OL tasks were processed based upon individual digit kinetics data, including: (1) digit normal force (Fn), which is the force component perpendicular to the grip surface used to grip on the object; (2) digit tangential force (Ft), which is the force component parallel to the grip surface to lift the object; and (3) digit center of pressure (CoP), which is the resultant force application point of each digit on the grip surface. We analyzed experimental variables as follows:

(1) Grip force $\left(\mathrm{F}_{\mathrm{G}}\right)$ was defined as the sum of Fn performed by all involved individual digits on the grip object.

(2) Tangential force $\left(\mathrm{F}_{\mathrm{T}}\right)$ was defined as the sum of $\mathrm{Ft}$ performed by all involved individual digits on the grip object. 


\section{MVC Task}

(3) Maximal grip force (Max_ $\mathrm{F}_{\mathrm{G}}$ ) denotes the peak value of $\mathrm{F}_{\mathrm{G}}$ over each trial in MVC task. Furthermore, a larger Max_ $F_{G}$ was selected from the two collected trials for each subject per digit condition.

(4) Digit force contribution in MVC task denotes the Fn produced by one, or a combination of digits when all involved digits reached $\mathrm{Max} \_\mathrm{F}_{\mathrm{G}}$.

\section{OL Task}

(5) Within-trial grip force adaptation $\left(\Delta \mathrm{F}_{\mathrm{G}}\right)$ was calculated as the difference between $\mathrm{F}_{\mathrm{G}}$ at the object lift onset and $\mathrm{F}_{\mathrm{G}}$ averaged during object hold. Our previous findings suggested a non-zero $\Delta \mathrm{F}_{\mathrm{G}}$ indicating a force adaptation resulting from an erroneous anticipatory control mechanism (Zhang et al., 2011).

(6) Object roll was defined as the absolute angle of deviation of the grip device from the vertical axis in the frontal plane. Maintaining the object orientation was required in OL task, and thus the peak object roll during object lift and averaged object roll during hold were used to determine subjects' performance as consequences of anticipatory force, moment control and the following adaptation respectively.

(7) Moment of forces. Individual digit moment of force (Mi) was calculated as the sum of the moment produced by Fn and the moment produced by $\mathrm{Ft}$ with respect to the center point of grip device "O" in the frontal plane (Figures 1A,B). Note that the total moment of forces $\left(\mathrm{M}_{\mathrm{TOT}}\right)$ applied to the device by all the involved digits should equal to zero to minimize object tilt in OL task.

(8) A Synergy index. We investigated how the digits coordinate with each other and quantified the multi-digit synergies based on a variance analysis (Latash et al., 2002; Zhang et al., 2009; SKM et al., 2012). An index $(\Delta V)$ of synergy was calculated as the normalized difference between the sum of the across-trial variances of elemental variables such as individual digit mechanical outputs and across-trial variance of the performance variable, i.e., the resultant output of the specified digits (Equation 1). In order to determine the multi-digit coordination at different hierarchies (Zhang et al., 2009), the synergy index was calculated at four levels: (a) hand individual digit level (Hand_d) involving T, I, M, $\mathrm{R}$ and $\mathrm{L}$ in $5 \mathrm{D}$, and $\mathrm{T}, \mathrm{I}, \mathrm{M}$ or $\mathrm{T}, \mathrm{R}, \mathrm{L}$ in $3 \mathrm{D}$; (b) thumbvirtual finger level $(T-V F)$ involving $\mathrm{T}$ and $\mathrm{VF}$ in both $5 \mathrm{D}$ and 3D; (c) VF individual finger level ( $V F \_f$ ) involving I, M, $\mathrm{R}$ and $\mathrm{L}$ in $5 \mathrm{D}$, and $\mathrm{I}, \mathrm{M}$ or R, L in 3D; and (d) VF anesthesia and non-anesthesia fingers level (VFa-VFna) involving I, $\mathrm{M}$ and $\mathrm{R}, \mathrm{L}$ in 5D only). At each hierarchical level, the multi-digit synergy was quantified with Equation 1 for three performance variables: normal force $\left(\Delta V_{-} F_{N}\right)$, tangential force $\left(\Delta V_{-} F_{T}\right)$ or resultant moment of forces $\left(\Delta V \_M\right)$ of specified digits.

$$
\Delta V \_P=\frac{\sum_{i} \operatorname{Var}\left(E_{i}\right)-\operatorname{Var} \sum_{i} E_{i}}{\sum_{i} \operatorname{Var}\left(E_{i}\right)}
$$

In Equation $1 E$ denotes the elemental variable (i.e., individual digit's Fn, Ft or M). $P$ denotes the performance variable studied (i.e., overall digits' Fn, Ft or $\mathrm{M}$ ), which can be calculated as $\sum_{i} E_{i}$. I denotes specified digits according to hierarchical levels. Var is the variance calculated across all 27 trials at two time epochs defined earlier (object lift onset and hold), for a given condition and grip type with first trial excluded. An emergence of a synergy stabilizing the resultant performance variable can be implied by positive values of $\Delta \mathrm{V}(\Delta V>0)$, which indicate negative co-variation (error compensation, Latash et al., 1998; Zhang et al., 2009) among elemental variables from trial to trial. Inversely, a negative $\Delta \mathrm{V}$ indicates an absence of multi-element synergy for the considered performance variable and level. The normalization by $\sum_{i} \operatorname{Var}\left(E_{i}\right)$ allows comparison across subjects and conditions.

\section{Martini Glass Task}

(9) Total water loss. The overall mass of water spilled over the three trials in martini glass task was evaluated to assess the overall task performance.

(10) Time spent. The duration of glass transportation of each trial was recorded to assess the task temporal performance.

\section{Statistical Analysis}

Multi-factor analysis of variance (ANOVA) with repeated measures were performed for experimental variables described above with the within-subject factors of Session (Anesthesia, Control), Grip type (5D, 3D_ane, 3D_mix in MVC task; 5D and 3D in OL task), Digit units (T, VFa, VFna), Phase (Lift onset, Hold), Hierarchical level (Hand_d, T-VF, VF_f,VFa-VFna), and Trial (1, 2, 3), and with the between-subject factor of Group (TIM group, TRL group).

In the MVC task, we performed a 3-way ANOVA with repeated measures on the Max_F $F_{G}$ with the factors of Session, Grip type and Group. Furthermore, the individual digit force contribution to the Max_ $\mathrm{F}_{\mathrm{G}}$ was evaluated for each Grip type by separate 3-way ANOVAs with repeated measures with factors of Session, Digit units and Group.

In the Lifting task, a preliminary test involving the factor of Trial (levels: 1, 2, .., 28) was performed for each individual experimental variable. Given that the first trial was the only trial found to be significantly different from the others, this trial of learning was omitted, and an average of data or variance of data was calculated based on subsequent trials (2-28) in further analysis. To investigate the subjects' performance with and without anesthetized digits, we performed a 3-way ANOVA with repeated measures on $\Delta \mathrm{F}_{\mathrm{G}}$ with factors of Session, Grip type and Group. In the aforementioned tests there was no main effect of Group nor interaction involving this factor, it was therefore removed from further statistical tests. Additionally, we performed a 3-way ANOVA with repeated measures on object roll with factors of Session, Grip type and Phase.

Given that the maximal value of synergy indices was limited by +1 , we applied Ficher's $z$-transformation to positive indices values before performing the statistics (Zhang et al., 2009) as 
follows: $\Delta \mathrm{V}=0.5(\ln (1+\Delta \mathrm{V})-\ln (1-\Delta \mathrm{V}))$. One-Sample $T$-tests were used to define whether each synergy index $\left(\Delta \mathrm{V}_{-} \mathrm{F}_{\mathrm{N}}, \Delta V \mathrm{~V}_{-} \mathrm{F}_{\mathrm{T}}\right.$ and $\left.\Delta \mathrm{V} \_\mathrm{M}\right)$ was different from zero. To determine the multi-digit coordination among anesthetized digits and/or non-anesthetized digits, we performed separate 3-way ANOVAs with repeated measures for each synergy index at each hierarchical level with the factors of Session, Grip type and Phase. In addition, to examine the multidigit coordination at different hierarchical control levels, we performed separate 3-way ANOVAs with repeated measures with factors of Session, Phase and Hierarchical level for each synergy index in $5 \mathrm{D}$ and $3 \mathrm{D}$ grip type individually, since Hierarchical level in 5D has four levels yet in 3D has three levels.

In the martini glass task, to investigate subjects' performance in a natural task with or without anesthetized digits, we performed a 2-way ANOVA with repeated measures on the total water loss with the factors of Session and Group, and a 3-way ANOVA with repeated measures on the time spent with Session, Trial and Group.

When the assumption of sphericity was violated, GreenhouseGeisser correction of degrees of freedom was used and thus the adjusted $P$ values were reported. Post hoc tests for pairwise comparisons were performed with Bonferroni adjustments when appropriate. The level of significance used was $P<0.05$. All reported values are averages across subjects \pm standard error of the mean.

\section{RESULTS}

All subjects in both anesthesia and control sessions were able to complete the experimental tasks as instructed, without dropping the device or showing fatigue during the experiment.

\section{MVC Task}

The averaged Max_F $F_{G}$ (Mean \pm SEM) across subjects under anesthesia and control sessions were plotted in Figure 2, for TIM (Figure 2A) and TRL (Figure 2B) groups separately. In MVC tasks, subjects exhibited significantly lower Max_ $F_{G}$ in the anesthesia session regardless of the grip type (main effect of Session: $\left.F_{(1,16)}=47.91, P<0.001\right)$. This observation is true for both groups of subjects no matter if subjects received anesthesia at TIM or TRL digits (no main effect of Group). Compared with the control session, maximal grip force was reduced by $48 \%$ in $5 \mathrm{D}, 44 \%$ in $3 \mathrm{D} \_$ane and $26 \%$ in $3 \mathrm{D} \_$mix for TIM group, $35 \%$ in $5 \mathrm{D}, 23 \%$ in $3 \mathrm{D} \_$ane, and $21 \%$ in 3D_mix for TRL group. In general, subjects were able to exert larger Max_ $\mathrm{F}_{\mathrm{G}}$ when using all the digits (5D) than three digits (3D_ane and 3D_mix) in the task (main effect of Grip type: $F_{(2,32)}=34.29$, $P<0.001)$. However, exceptions were found in the anesthesia session showing similar Max_F $F_{G}$ between 5D and 3D_ane in TIM group, and similar Max_ $\mathrm{F}_{\mathrm{G}}$ between 5D and 3D_mix in TRL group (interaction effect of Session $\times$ Grip type $\times$ Group: $F_{(2,32)}$ $=8.39, P<0.001)$. Furthermore, Max_ $\mathrm{F}_{\mathrm{G}}$ produced by the three selected digits (3D_ane) was larger compared with that by the other three-digit combination (3D_mix) before anesthesia in the control session, but turned out to be smaller in the anesthesia

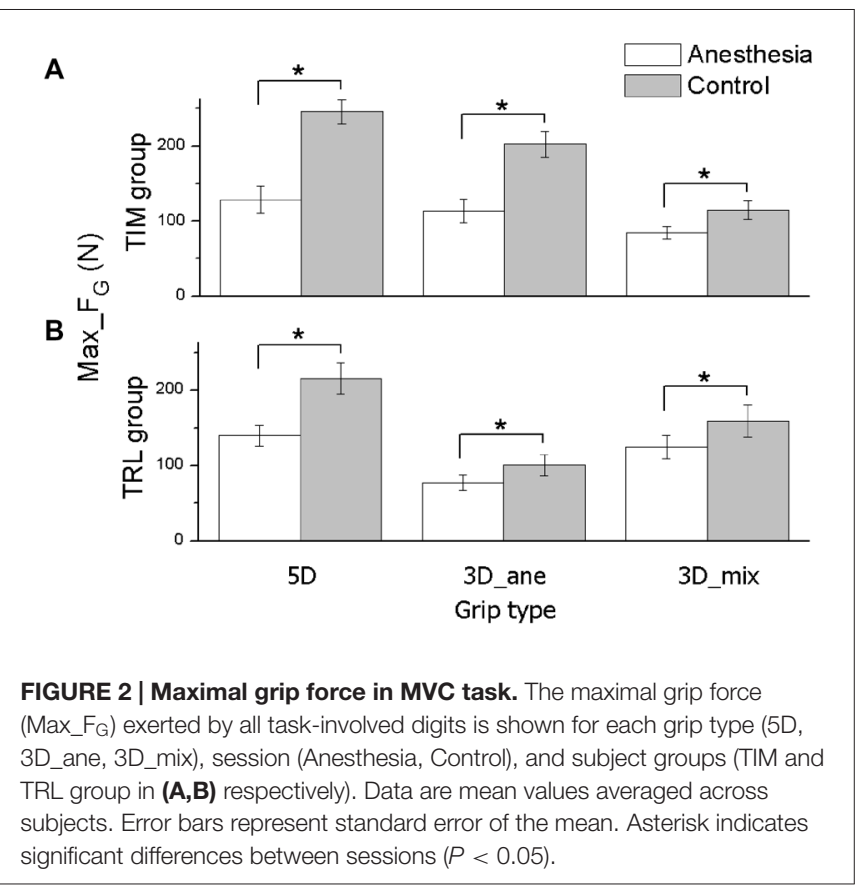

session (interaction effect of Session $\times$ Grip type: $F_{(2,32)}=28.77$, $P<0.001)$. The different Max_ $\mathrm{F}_{\mathrm{G}}$ observed in two 3D grip conditions was also varied in two subject groups (interaction effect of Grip type $\times$ Group: $\left.F_{(2,32)}=21.98, P<0.001\right)$. Post hoc tests revealed a larger Max_F $F_{G}$ for TIM group, yet a smaller Max_ $F_{G}$ for TRL group in 3D_ane compared with 3D_mix grip condition.

In order to identify whether the overall Max_ $F_{G}$ decrease presented in anesthesia session was introduced by the anesthetized digits or not, we plotted the averaged Fn contribution in MVC tasks from the thumb (T), VFa and VFna across subjects in anesthesia and control sessions for each grip condition (Figure 3). In fact, all the digits exerted significantly less force to reach the Max_F $F_{G}$ after the anesthesia injection, including VFna, for which the Fn production dropped by $20.91 \pm 0.9 \mathrm{~N}$ in $5 \mathrm{D}$ and $15.4 \pm 1.61 \mathrm{~N}$ in 3D_mix grip conditions from control to anesthesia session (main effect of Session: $F_{(1,16)}=52.64,59.51$, and 12.36 in 5D, 3D_ane and 3D_mix, respectively; $P<0.005)$. In addition, digit units contributed to the Max_ $\mathrm{F}_{\mathrm{G}}$ differently. Specifically in $5 \mathrm{D}$, thumb contributed the most when compared to both VFa and VFna in each session (main effect of Digit units: $F_{(2,32)}=124.09$, $P<0.001$ ) while VFa exerted larger Fn in the control session but less Fn in the anesthesia session than VFna (interaction effect of Session $\times$ Digit units: $\left.F_{(2,32)}=14.43, P<0.001\right)$. In addition, subjects in TIM group contributed more Fn from VFa than VFna, whereas subjects in TRL group presented the opposite (Digit units $\times$ Group: $\left.F_{(2,16)}=17.48, P<0.001\right)$. No significant difference was observed between the two subject groups (no main effect of Group), except in 3D_ane grip condition, subjects in TIM group produced larger force than TRL group during the control session (Session $\times$ Group interaction: $F_{(1,16)}=20.40$, $P<0.001)$. 


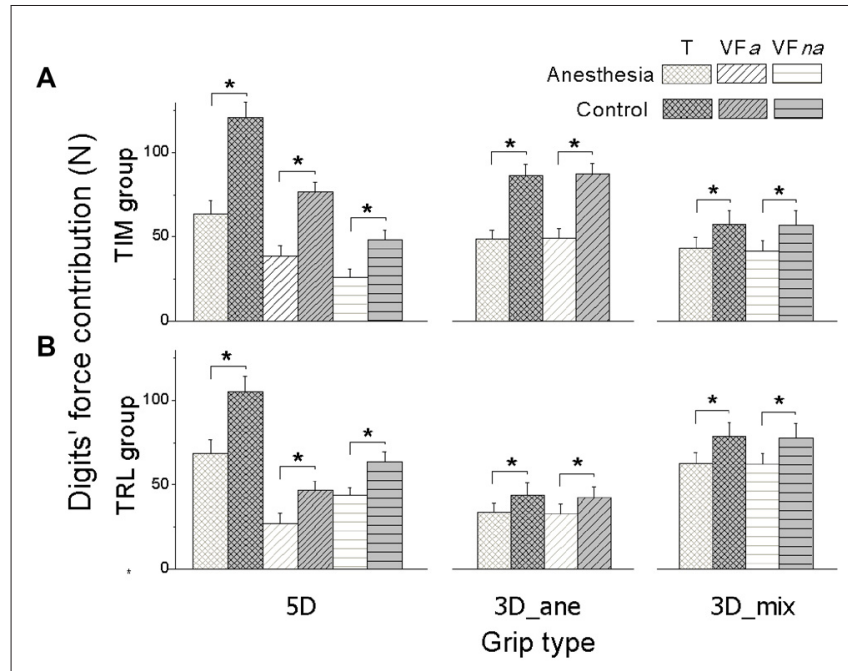

FIGURE 3 | Digits' force contribution in MVC task. Normal force contribution from the thumb (T), anesthetized virtual fingers (VFa) and non-anesthetized virtual fingers (VFna) when reaching the maximal grip force is shown for each grip type (5D, 3D_ane, 3D_mix), session (Anesthesia, Control), and subject groups (TIM and TRL group in $(\mathbf{A}, \mathbf{B})$ respectively). Data are mean values averaged across subjects. Error bars represent standard error of the mean. Asterisk indicates significant differences between sessions $(P<0.05)$.

\section{OL Task Performance}

Note that trial 1 was omitted, given the significant difference between the first and the subsequent trials. Thus, the results reported in OL task were based on averages across trial 2-28 (see data analysis). In OL task, subjects were asked to lift the object and keep it as vertical as possible. To evaluate subjects' performance of object grasping and lifting, grip force and peak object roll were quantified in the following. Given no significant difference found between the two subject groups (TIM vs. TRL) for all the experimental variables regardless the grip types (no main or interaction effect of Group), data plotted in the following were therefore averaged over two groups for simplicity.

Figure 4 showed the averaged $\mathrm{F}_{\mathrm{G}}$ at object lift onset as well as during object hold across subjects in anesthesia and control sessions by using all five digits $(5 \mathrm{D})$ or three anesthetized digits (3D). In general, subjects dropped $F_{G}$ production from object lift onset to object hold. However, such grip force decrease $\left(\Delta \mathrm{F}_{\mathrm{G}}\right)$ in anesthesia and control sessions were dependent on the grip types (interaction Session $\times$ Grip type: $F_{(1,17)}=14.48, P<0.05$ ). Specifically, similar $\Delta \mathrm{F}_{\mathrm{G}}$ was observed in both anesthesia $(4.70 \pm$ $0.73 \mathrm{~N})$ and control $(4.53 \pm 0.81 \mathrm{~N})$ sessions by using 3D grip, whereas subjects showed significantly less $\Delta \mathrm{F}_{\mathrm{G}}$ in anesthesia $(2.77 \pm 0.80 \mathrm{~N})$ than in the control session $(7.64 \pm 1.53 \mathrm{~N})$ by using 5D grip $(P<0.05)$.

We plotted in Figure 5 the maximal object roll during $\mathrm{OL}$ and averaged object roll over hold phase for $5 \mathrm{D}$ and $3 \mathrm{D}$ grip conditions, averaged across subjects in anesthesia and control sessions. We observed a general larger object roll in the anesthesia (during lifting: $2.56 \pm 0.22^{\circ}$; during hold: 1.78 $\pm 0.24^{\circ}$ ) than in the control session (during lifting: $1.93 \pm 0.08^{\circ}$; during hold: $0.95 \pm 0.08^{\circ}$ ) at both time epochs (main effect of

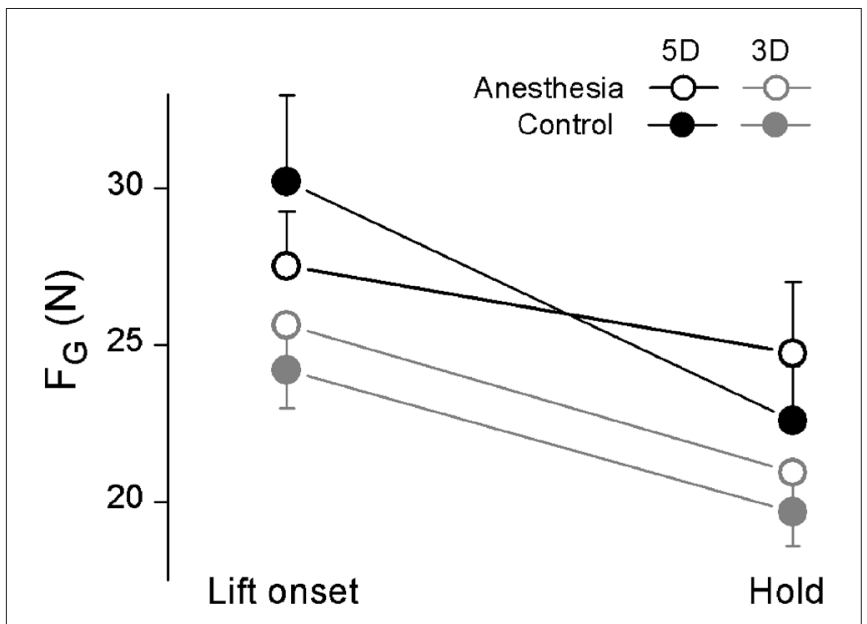

FIGURE 4 | Grip force at object lift onset and during object hold in OL task. The grip force $\left(\mathrm{F}_{\mathrm{G}}\right)$ measured at lift onset (left) and during hold (right) is shown each grip type (5D and $3 \mathrm{D}$ ) and session (Anesthesia, Control). Data are mean values averaged across trials two through 28 and further across subjects in both TIM and TRL groups. Error bars represent standard error of the across-subject mean.

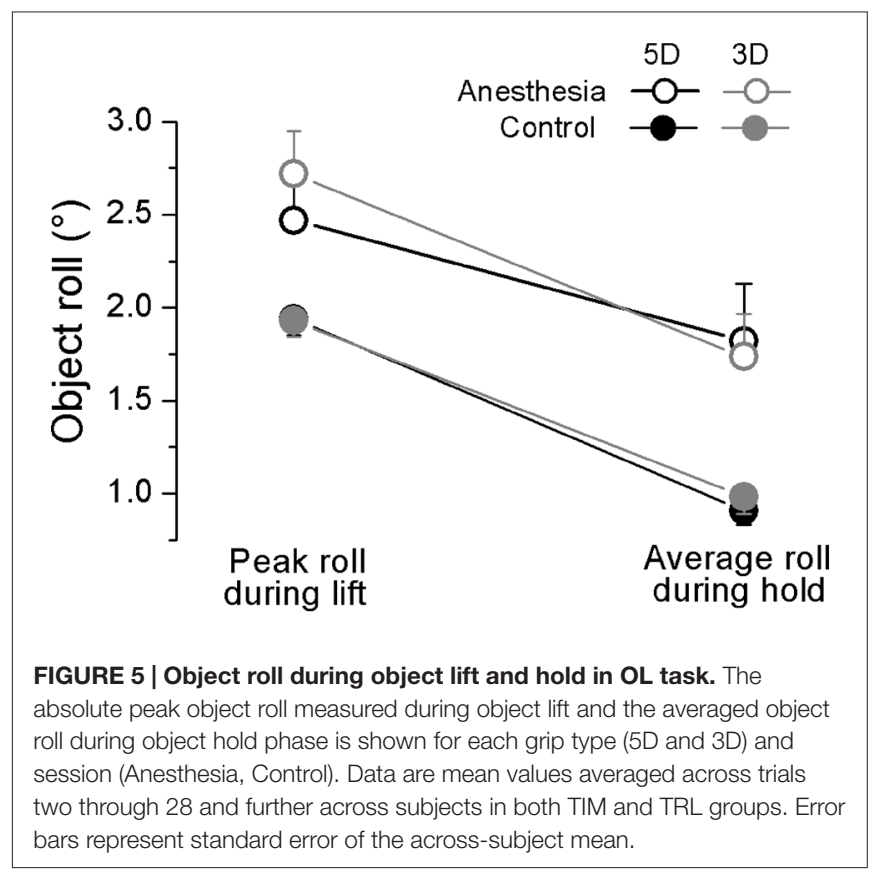

Session: $\left.F_{(1,17)}=10.56, P<0.01\right)$, except during hold using $5 \mathrm{D}$ (Session $\times$ Grip type $\times$ Phase interaction: $\left.F_{(1,17)}=6.81, P<0.05\right)$. Additionally, all subjects generated significantly larger object roll during lift than hold phase (effect of Phase: $F_{(1,17)}=138.46$, $P<0.001)$.

\section{Multi-Digit Coordination in OL Task}

As previously stated, the synergy indices stabilizing the normal force, tangential force as well as the moment of force in the object frontal plane have been calculated at four different hierarchical levels: Hand_d, T-VF, VF_f and VFa-VFna. We 
plotted $\Delta V_{-} F_{N}, \Delta V_{-} F_{T}$, and $\Delta V \_M$ averaged across subjects within the anesthesia and control sessions at object lift onset and hold in Figures 6-8 respectively. As shown in Figure 6, different $\triangle \mathrm{V} \_\mathrm{F}_{\mathrm{N}}$ values were observed at four hierarchical levels (main effect of Hierarchical level: $F_{(1.08,17.32)}=375.16$ in $5 \mathrm{D} ; F_{(1.00,16.00)}$ $=1341.0$ in $3 \mathrm{D}$; both $P<0.001)$. Specifically, subjects presented high values of $\Delta V_{-} F_{N}(+1)$ at both levels involving the thumb (Hand_d and T-VF) regardless of the sessions, phases or grip types. In contrast, all subjects showed negative values of $\Delta V_{-} F_{N}$ at levels involving only VF fingers ( $V F \_f$ and $\left.V F a-V F n a\right)$ : $t$-value within the range of $(-9.676 ;-2.260)$ across individual comparisons; all $P<0.05$. Given the absence of synergy, $\Delta \mathrm{V} \_\mathrm{F}_{\mathrm{N}}$ was not investigated further.

As plotted in Figure 7, $\Delta \mathrm{V}_{-} \mathrm{F}_{\mathrm{T}}$ was smaller by 0.1 in the anesthesia compared with the control session, even though the

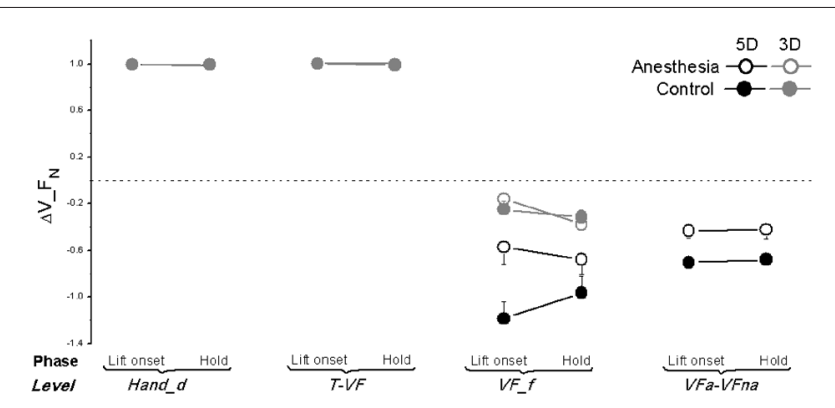

FIGURE 6 | Normal force synergy indices in OL task. The synergy index quantifying multi-digit coordination of normal force output $\left(\Delta V_{-} F_{N}\right)$ is calculated at object lift onset and during object hold and shown for each grip types, session and hierarchical level (Hand_d: individual digits of the hand, $T-V F$, thumb vs. virtual finger; $V F \_f$, individual fingers of the virtual finger; VFa-VFna, anesthetized vs. non- anesthetized virtual fingers). A dotted line denotes a zero line to distinguish positive (synergy presence) and negative (no synergy presence) $\Delta \mathrm{V}$ indices. Data are mean values averaged across subjects in both TIM and TRL groups. Error bars represent standard error of the across-subject mean.

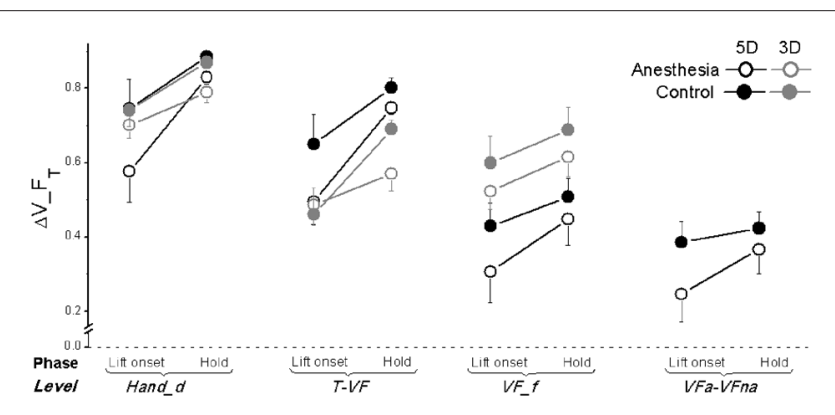

FIGURE 7 | Tangential force synergy indices in OL task. The synergy index quantifying multi-digit coordination of tangential force output $\left(\Delta V_{-} F_{T}\right)$ is calculated at object lift onset and during object hold and shown for each grip types, session and hierarchical level (Hand_d: individual digits of the hand, $T-V F$, thumb vs. virtual finger; $V F \_f$, individual fingers of the virtual finger; VFa-VFna, anesthetized vs. non- anesthetized virtual fingers). A dotted line denotes a zero line to distinguish positive (synergy presence) and negative (no synergy presence) $\Delta \mathrm{V}$ indices. Data are mean values averaged across subjects in both TIM and TRL groups. Error bars represent standard error of the across-subject mean.

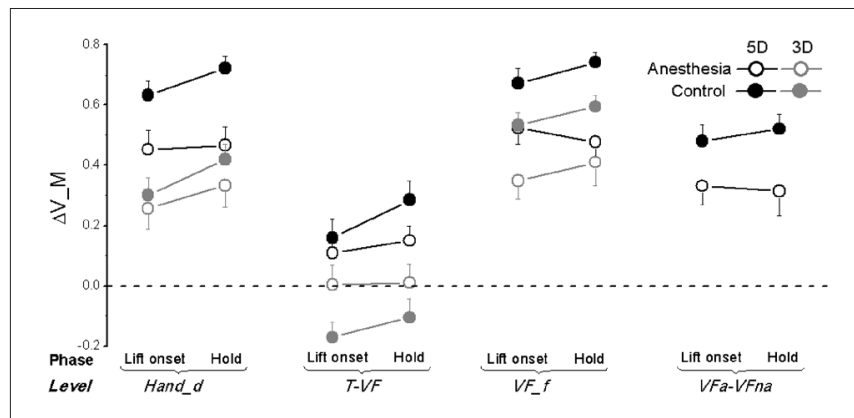

FIGURE 8 | Moment of force synergy indices in OL task. The synergy index quantifying multi-digit coordination of moment of force output $\left(\Delta V_{-} M\right)$ is calculated at object lift onset and during object hold and shown for each grip types, session, and hierarchical level (Hand_d: individual digits of the hand, T-VF, thumb vs. virtual finger; VF_f, individual fingers of the virtual finger; VFa-VFna, anesthetized vs. non- anesthetized virtual fingers). A dotted line denotes a zero line to distinguish positive (synergy presence) and negative (no synergy presence) $\Delta \mathrm{V}$ indices. Data are mean values averaged across subjects in both TIM and TRL groups. Error bars represent standard error of the across-subject mean.

observed discrepancy between the sessions was significant only at the level of Hand_d (main effect of Session: $F_{(1,17)}=14.23$; $P<0.05$ ) and T-VF (main effect of Session: $F_{(1,17)}=6.02$; $P<0.05)$. In addition, significantly lower $\Delta V_{-} F_{T}$ was observed at object lift onset than object hold (main effect of Phase: $F_{(1,17)}$ $=31.23$ for Hand_d, $F_{(1,17)}=33.40$ for T-VF, $F_{(1,17)}=7.47$ for $V F \_f$; all $\left.P<0.05\right)$, except at $V F a-V F n a$ level. Moreover, subjects altered their synergy index of tangential force when using different grip types: at the level of T-VF, a significantly

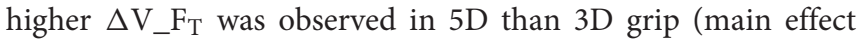
of Grip type: $\left.F_{(1,17)}=1.81, P<0.001\right)$; at the level of $V F \_f$, however, $\Delta \mathrm{V}_{-} \mathrm{F}_{\mathrm{T}}$ was significantly lower in $5 \mathrm{D}$ than $3 \mathrm{D}$ grip (main effect of Grip type: $F_{(1,17)}=17.69, P<0.005$ ). Furthermore, $\triangle \mathrm{V}_{-} \mathrm{F}_{\mathrm{T}}$ showed varied values at different hierarchical levels (main effect of Hierarchical level: $F_{(1.3,20.80)}=53.14$ in 5D; $F_{(1.04,16.58)}=20.26$ in $3 \mathrm{D}$; both $\left.P<0.001\right)$. For both grip types, subjects presented the highest $\Delta \mathrm{V}_{-} \mathrm{F}_{\mathrm{T}}$ at the level of Hand_d. Additionally in $5 \mathrm{D}$ grip, the lowest $\Delta \mathrm{V}_{-} \mathrm{F}_{\mathrm{T}}$ was observed at the level of $V F a-V F n a$.

Similar to $\Delta \mathrm{V}_{-} \mathrm{F}_{\mathrm{T}}$, subjects also exhibited lower values of $\Delta \mathrm{V} \_\mathrm{M}$ after digital anesthesia $(0.32 \pm 0.05)$ compared with controls $(0.43 \pm 0.08)$ as shown in Figure 8. This observation is significant for level of $V F \_f$ and $V F a-V F n a$ (main effect of Session: $F_{(1,17)}=17.38$ and 10.47 , respectively; both $P<0.01$ ) as well as for level of Hand_d but only for 5D grip condition (interaction effect of Session $\times$ Grip type: $F_{(1,17)}=6.66$, $P<0.05)$. Not surprisingly, subjects increased their synergy index of $\Delta \mathrm{V} \_\mathrm{M}$ during hold from object lift onset, which were significant for levels of Hand_d and T-VF (main effect of Phase: $F_{(1,17)}=8.31$ and 6.10 , respectively; both $\left.P<0.05\right)$. In addition, we also observed varied $\Delta V_{\_} M$ by using different grip types. Particularly, subjects showed significantly higher values in $5 \mathrm{D}$ than 3D at individual digit levels, i.e., Hand_d and VF_f (main effect of Grip type: $F_{(1,17)}=23.77$ and 12.37, respectively; both $P<0.005)$, as well as at T-VF level, but in the control session only (interaction Session $\times$ Grip type: $F_{(1,17)}=4.95, P<0.05$ ). 
Moreover, subjects presented different $\Delta \mathrm{V} \_\mathrm{M}$ calculated at different hierarchical levels for both $5 \mathrm{D}$ and 3D grip types (main effect of Hierarchical Levels: $F_{(1.22,19.56)}=74.12$ in $5 \mathrm{D}, F_{(1.21,19.29)}$ $=90.31$ in $3 \mathrm{D}$; both $P<0.001)$. We observed $\Delta \mathrm{V} \_\mathrm{M}$ values at $\mathrm{T}-\mathrm{VF}$ level were the smallest of all levels regardless of grip types, and furthermore, for 3D grip type $\Delta \mathrm{V} \_\mathrm{M}$ was significantly larger at $V F \_f$ level than at Hand_d level for subjects in the control session only (interaction Session $\times$ Level: $F_{(2,32)}=11.20$, $P<0.05)$.

\section{Martini Glass Task}

For the martini glass task, we plotted the averaged subjects' martini glass task performance, i.e., total water loss and duration of transportation, for both the anesthesia and control sessions in Figure 9. As seen from Figure 9A, subjects spilled more water in the anesthesia $(82.89 \pm 12.96 \mathrm{~g})$ compared with the control session $(52.28 \pm 4.97 \mathrm{~g})$ regardless of the group (main effect of Session: $F_{(1,16)}=6.44, P<0.05$ ). Figure 9B shows that, during the first trial, subjects in the anesthesia session spent more time to transport the martini glass filled with water between two instructed locations (anesthesia: 22.19 $\pm 2.15 \mathrm{~s}$; control: $18.36 \pm 1.40 \mathrm{~s}$ ). However, in subsequent trials (trial 2 and trial 3), subjects throughout both sessions completed the task with similar duration of glass transportation (interaction Session $\times$ Trial: $F_{(2,32)}=3.76, P<0.05$ ). Subjects in both sessions showed a decrease of transportation duration from the first $(20.3 \pm 1.7 \mathrm{~s})$ to the second trial $(16.7 \pm$ $1.4 \mathrm{~s}$; main effect of Trial: $\left.F_{(2,32)}=23.86, P<0.001\right)$. No further decrease was observed from the second $(15.6 \pm 1.5$ $\mathrm{s})$ to the third trial $(14.8 \pm 1.7 \mathrm{~s})$ in the control session, however, subjects in the anesthesia session continued with shortening time in the subsequent trials (Trial 2: $17.8 \pm 1.7 \mathrm{~s}$; Trial 3: $15.4 \pm 1.9 \mathrm{~s} ; P<0.005)$. No main or interaction effect of Group was found in these martini glass task performance variables.
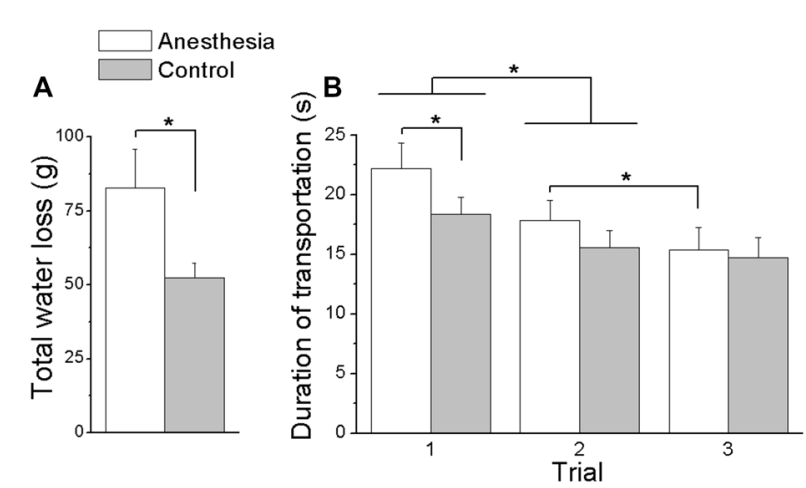

FIGURE 9 | Total water loss and duration of transportation in Martini glass task. (A) Shows the overall water spilled cumulated over three trials in the Martini glass task for both anesthesia and control sessions. (B) Shows the duration of transportation in the Martini glass task for each trial and session. Data are mean values averaged across subjects in both TIM and TRL groups. Error bars represent standard error of the across-subject mean. Asterisk indicates significant differences between sessions $(P<0.05)$.

\section{DISCUSSION}

\section{Effect of Local Anesthesia on Digital Maximal Grip Force Abilities}

In MVC tasks, subjects showed decreased maximal force application in grasping after digital anesthesia injections (Figure 2). This result is consistent with previous reports in pinch conditions (Augurelle et al., 2003) as well as in individual or four-finger isometric pressing tasks (Shim et al., 2012; Kim et al., 2013). These findings indicate that the deficiency of sensory feedback would reduce the MVC ability in the digits. The force reduction induced by local anesthesia, may be due to decreased cortical motoneuron excitability after sensory flow is altered. For example, Rossini et al. (1996) found that anesthesia of the median and radial nerves induced a decrement of MEPs amplitude in first dorsal interosseous muscle. Nevertheless, experimental sets up for earlier findings have been limited to the local (anesthetized) digits, thus overlooked the interactive adaptations among local and non-local (non-anesthetized) digits. Our current results revealed that the deficit in maximal force production presented not only on deafferent digits but also on intact digits.

First, the significant drop in Max_ $\mathrm{F}_{\mathrm{G}}$ appeared in all three grip types, i.e., anesthetized thumb opposing two anesthetized and two non-anesthetized fingers, anesthetized thumb opposing two anesthetized fingers, and anesthetized thumb opposing two non-anesthetized fingers. Since the thumb is the only digit on its side in the grasp, opposing the other fingers, it mechanically contributes $50 \%$ to the overall grip force. Therefore, given thumb is the only digit systematically anesthetized throughout the grip types, the thumb force deficit (Li et al., 2004) could constrain the overall Max_F $F_{G}$ amplitude, leading to a general grip force drop. However, this is true only when the thumb is physically weaker than its opposing fingers, such as in $5 \mathrm{D}$. The removal of somatosensory feedback from three digits resulted in similar Max_ $\mathrm{F}_{\mathrm{G}}$ regardless if subjects used all five digits or three anesthetized digits (i.e., T, I and M). That is, adding two more fingers ( $\mathrm{R}$ and $\mathrm{L}$ ) in the grip didn't raise the overall grip force in the anesthesia session as it did in the controls, despite the potential intact sensation input from the added fingers (Figure 2, TIM group). Despite this, the post-anesthesia thumb-constrained Max_. $\mathrm{F}_{\mathrm{G}}$ was not applied in all the grip types, since the amplitude of Max_ $\mathrm{F}_{\mathrm{G}}$ was not consistent across grip types in anesthesia session (Figure 2). The lowest Max_F $F_{G}$ and thus the thumb Fn was produced by enrolling thumb, ring and little fingers in the MVC task (i.e., 3D_mix in TIM group, 3D_ane in TRL group). In these conditions, Max_F $F_{G}$ was most likely constrained by the VF Fn production (in $\mathrm{R}$ and $\mathrm{L}$ ) due to its weaker absolute strength compared to the thumb. These observations lead to a very interesting finding, in that, the digital anesthesia in local digits also affected the $\mathrm{MVC}$ in the non-local digits, inducing the maximal grip force disability.

Further results in digits' force contribution showed a universal Fn drops in the anesthesia session from all the taskinvolved digits, including the non-anesthetized digits in $5 \mathrm{D}$ (Figure 3). These novel findings showed that the effect of digital 
anesthesia on the maximal ability in grip force production is grip type-dependent based upon local and non-local fingers involvement. The absent somatosensory feedback induced by selective digital anesthesia led to impairment in the MVC at both local and non-local digits, resulting in subjects' reduced ability in maximal grip force production. Local and non-local responses have been examined in some earlier studies during submaximal grip force tasks in healthy hands. Aoki et al. (2007) investigated the role of tactile information in adapting grip object properties, and found that changes in texture at a given digit elicit force adjustments at the same as well as other digits, indicating that sensory information at one digit affects the force modulation at non-stimulated digits. Our current results further implied that individual sensory input is shared across all the digits and the disturbed signal from local sensory channel(s) has a more comprehensive impact on the process of the motor output execution in the sensorimotor integration.

\section{Absent Digital Sensation Induced Deficits in Force and Moment Control}

In object lift task, the subjects didn't present observable erroneous actions such as dropping the device after selective digital anesthesia. In particular, subjects were able to exert sufficient $F_{G}$, regardless the involved digits were all anesthetized (3D) or selectively anesthetized (5D). Furthermore, subjects presented relative similar $\mathrm{F}_{\mathrm{G}}$ before and after digital anesthesia at both lift onset and during hold (Figure 4). These observations suggest that capabilities in grip force control can be preserved even if the somatosensory information is disturbed. This may be because the residual sensory input available in the hand and/or forearm was recruited to assist the motor output in the sensorimotor integration process. Note that our results didn't show an excessively large $\mathrm{F}_{\mathrm{G}}$ as reported in earlier studies after nerve block at either digital level (Johansson and Westling, 1984; Monzée et al., 2003) or lower median nerve level (Dun et al., 2007). The discrepancy between the current and previous results might due to the different experimental designs. First, previous studies have been limited to pinch grip with both involved digits anesthetized. Second, the objects used in their lift task were much lighter (varied from $50 \mathrm{~g}$ to $400 \mathrm{~g}$ ) than in the current study (665 g). Three-digit grasping has been explored by Nowak et al. (2001), but during a dynamic oscillation not steady hold task. We suspect that the sensory information originated from skin deformation could enhance the residual proprioception (Edin and Johansson, 1995) in the hand and/or the forearm with increased cutaneous strain when lifting a much heavier object. Recent studies have investigated the effect of digital sensory deficit introduced by CTS on grasp control. They found a remarkable larger grip force compared with healthy controls in $5 \mathrm{D}$ involving both affected and non-affected digits, but not in $2 \mathrm{D}$ or $3 \mathrm{D}$ when all involved digits were affected (Zhang et al., 2011, 2012, 2013). Such grip force increase in CTS patients during the whole hand grasp might be a strategic adaptation in force control as a result from erroneous actions in daily life activities, such as frequent object drops. However, the digital anesthesia applied in our study artificially removed the tactile and proprioception input from the digits in a temporary fashion. In contrast to the patients who suffered tactile sensory impairment gradually and chronically, our subjects had no explicit preknowledge regarding their sensory deficiency and consequential motor output.

Despite the residual ability of grip force control discussed above, our results also revealed subtle behavioral inefficiencies in the whole hand grasp while digital sensory inputs were selectively blocked. Grip force is exerted at the time of lift onset, following anticipatory control fashion (Fu et al., 2010). Therefore, the general decrease in $\mathrm{F}_{\mathrm{G}}$ from lift onset to the object holding phase implies a correction from an overshoot output based on feedforward control mechanism. This correction results in a more efficient force pattern (i.e., closer to taskrequired minimum force) when online feedback information is utilized to determine the behavioral performance (e.g., object roll), such as, visual or residual somatosensory sensory input from the hand and/or forearm. In this scenario, the $F_{G}$ drop between two time epochs reflects an ability of sensorimotor adaptation responsible to object property (i.e., weight). Our results showed that subjects maintained the within-trial force adaptation ability in 3D but not in 5D after three digits' anesthesia (Figure 4). This is an interesting yet counterintuitive finding, since utilizing all anesthetized digits lead to "normal" force adaptation, but recruiting non-anesthetized digits with intact sensory output resulted in a deficit of force control. This result prompted a central processing mechanism in the sensorimotor integration, that is, the accurate input available from intact sensory channels is not prioritized over the disturbed sensory input when both coexist. Instead, the central controller will integrate all the sensory signals with varied precision, and utilize the processed information for updating the taskrelevant motor program execution. This assumption imposes a larger challenge to CNS in a motor task when recruiting mixed sensory signals with unequally disturbed perception compared to a uniformly impaired sensory system. This assumption is in accordance with our previous study on CTS patients, which demonstrated an inefficient grip force production when adding intact digits in the grip, but not for sensory-impaired digits only (Zhang et al., 2013).

In addition, subjects presented with a decreased ability to maintain the object's vertical orientation during lift or transportation. Our results showed that removing the cutaneous and proprioception sensory information led to a larger object roll during object lift as a result of anticipatory control and object hold following the online motor adaptation control (Figure 5). Similar abated performance was been observed in $3 \mathrm{D}$ and $5 \mathrm{D}$, indicating the intact sensory channels available from non-anesthetized digits in 5D were not capable to help to improve the task performance that was induced by impaired sensory channels. Correspondingly, subjects' deficit in moment control was also reflected in the daily life task (i.e., martini glass transportation), as evidenced by the larger amount of water spill and slower transportation speed after digital anesthesia (Figure 9). These results manifest the difficulty in moment control elicited by sensory deficiency in grasping manipulative 
tasks. In studies when resultant moment was not required to be maintained (e.g., no need to keep the object vertical orientation), or when the feedback on object roll induced by erroneous moment production was not provided (e.g., object was physically constrained and allows no tilt), an inefficient moment production was observed in a pinch grip after digital anesthesia (Monzée et al., 2003). In our current study, the object tilting and water spilling could be evaluated as the erroneous output based on visual and/or residual sensory feedback from the hand and the forearm. In this scenario, subjects' failure of accurate moment production indicates that residual sensory information is not sufficient to assist the motor correction in moment control process.

\section{Feedback Control Component Resides in Motor Synergy Structure}

Very few studies have determined the multi-digit coordination patterns underneath digital anesthesia conditions. Koh et al. (2015) investigated motor synergies based on the force acrosstrial variability among four fingers in isometric pressing tasks. As a result, there was no synergy drop identified in the absence of digital sensory feedback. The reported preserved synergy might due to the dominant role of visual feedback in accurate force production and correction compared with cutaneous sensory modality (Hartcher-O'Brien et al., 2008; Touzalin-Chretien et al., 2010). Our current study examined the effect of digital anesthesia on multi-digit coordination in object grasp and lift control. Most importantly, we quantified the multi-digit synergy index (reviewed in Latash et al., 2003) responsible for task-specific force and moment control at different hierarchy levels involving digits with and without sensory deficiency. One general result is that multi-digit motor synergies were presented (i.e., $\Delta \mathrm{V}>0$ ) in stabilizing the total or subtotal Fn (Figure 6), $\mathrm{F}_{\mathrm{T}}$ (Figure 7) or the moment in frontal plane (Figure 8), yet to different extents and at different levels. Even though the nearly perfect synergy index value (close to 1) was observed for Fn, indicating a high covariation structure across the individual force output among digits (Hand_d) as well as between thumb and virtual finger $(T-V F)$, it was not given much attention. This is because the "perfect" coordination of Fn by the thumb and fingers was artificially formulated by the task mechanics, i.e., the Fn produced by the thumb should be equal but opposite to that exerted by VF in object lift task. However, when the force coordination was not mandated among the digits within VF side (i.e., at the level of $V F \_f$ and $V F a-V F n a$ ), synergy disappeared in both control and anesthesia sessions. This suggests that the subtotal normal force from the involving fingers is not intentionally stabilized and controlled from the central level in hand grasp control task.

Different from normal force, we found synergistically motor coordination patterns for both tangential force or moment outputs across the task-involved digits. Grasp task has been viewed from two-subtask perspectives: object lift and its orientation maintenance. The parallel presence of $\mathrm{F}_{\mathrm{T}}$ synergy and moment synergy implies a superposition principle of force control and moment control in object grasping task (Zatsiorsky et al., 2004). The general observed positive values of $\Delta V_{-} F_{T}$ and $\Delta \mathrm{V} \_\mathrm{M}$ at different levels imply a covariation structure among the elemental variables, revealed as the mechanical outputs from different hierarchical control levels involving either the subsets of digits (e.g., T-VF and VFa-VFna) or the individual digits (e.g., Hand_d and VF_f). The observed higher $\Delta \mathrm{V}$ indices at levels of Hand_d and VF_f for both synergies complies with an earlier theoretical assumption, which is, synergies co-exist and are lack of interference (Zhang et al., 2008). This finding further indicates a parallel but independent process of tangential force and moment stabilization, which was mostly achieved by covariation at the individual digits level. Particularly, the overall $\mathrm{F}_{\mathrm{T}}$ was stabilized primarily by coordinating $\mathrm{Ft}$ across individual digits including the thumb (i.e., highest $\Delta \mathrm{V}_{-} \mathrm{F}_{\mathrm{T}}$ at level of Hand_d). The resultant moment, however, was stabilized mainly by coordinating the moment from individual fingers at the VF side (i.e., highest $\Delta \mathrm{V}_{-} \mathrm{M}$ at level of VF_f). The discrepancy of effector involvement between the synergies may relate to the different role of the thumb in force vs. moment control. The former controller requires upward Ft production from the thumb as well as from the other digits, whereas the latter controller may view the thumb as the pivot point for moment production by other fingers.

Most importantly, the presented force or moment synergies were affected and weakened by selective digital sensory blocks. The attenuated motor synergies present underneath digital anesthesia reveals a feedback control component in formation process for the motor output covariation structure. Consequently, a defective moment coordination pattern induced by digital anesthesia was embodied in notable behavioral features, such as object roll. The role of sensation feedback in motor synergy structure has been discussed controversially in different schemes or models. For example, feedback signals have been proposed to assist coordinating output signals of finger forces in optimal control schemes (Todorov and Jordan, 2002) and in a model based on action of central back-coupling loops (Latash et al., 2005). Contrarily, a feed-forward scheme suggested that the formation of motor variabilities structure may not consist any explicit feedback correction mechanisms (Goodman and Latash, 2006). The problem is that these computational models need to be testified in experimental protocols that allow eliminating or altering sensory feedback input, different digits' enrollment, and accurate moment control. Our study emphasized that a sensory deficit at a subset of digits impaired the motor coordination patterns among all involved digits. The influential effect of digital anesthesia on task-specific motor synergies corroborated the favorable mechanism of feedbackbased coordination of the elemental motor variables, indicating a peripheral sensory deficit induced changes in the CNS control mechanism. This hypothetical control mechanism needs further investigations in more moment control tasks.

\section{CONCLUSION}

In summary, our results suggested that the absence of somatosensory information induced motor deficits in hand grasp control, as evidenced by reduced maximal force production 
ability in both local and non-local digits, impairment of force and moment control in object lift and hold, and attenuated motor synergies in stabilizing task performance variables, namely the tangential force and moment of force. These findings implied that individual sensory input is shared across all the digits and the disturbed signal from local sensory channel(s) has a more comprehensive impact on the process of the motor output execution in the sensorimotor integration process. Additionally, a feedback control mechanism with a sensory-based component resides in the formation process for the motor covariation structure.

\section{AUTHOR CONTRIBUTIONS}

WZ contributed to the design of the study. AC, WZ, KM, CG, ER and MT contributed to subject recruitment and data acquisition.

\section{REFERENCES}

Abbruzzese, G., and Berardelli, A. (2003). Sensorimotor integration in movement disorders. Mov. Disord. 18, 231-240. doi: 10.1002/mds.10327

Aoki, T., Latash, M. L., and Zatsiorsky, V. M. (2007). Adjustments to local friction in multifinger prehension. J. Mot. Behav. 39, 276-290. doi: 10.3200/jmbr.39.4. 276-290

Arbib, M. A., Iberall, T., and Lyons, D. (1985). Coordinated control programs for movements of the hand. Exp. Brain Res. Suppl, 10, 111-129. doi: 10.1007/9783-642-70105-4_7

Augurelle, A. S., Smith, A. M., Lejeune, T., and Thonnard, J. L. (2003). Importance of cutaneous feedback in maintaining a secure grip during manipulation of hand-held objects. J. Neurophysiol. 89, 665-671. doi: 10.1152/jn.00249. 2002

Baud-Bovy, G., and Soechting, J. F. (2001). Two virtual fingers in the control of the tripod grasp. J. Neurophysiol. 86, 604-615.

Dun, S., Kaufmann, R. A., and Li, Z. M. (2007). Lower median nerve block impairs precision grip. J. Electromyogr. Kinesiol. 17, 348-354. doi: 10.1016/j.jelekin. 2006.02.002

Edin, B. B., and Johansson, N. (1995). Skin strain patterns provide kinaesthetic information to the human central nervous system. J. Physiol. 487, 243-251. doi: 10.1113/jphysiol.1995.sp020875

Fellows, S. J., Noth, J., and Schwarz, M. (1998). Precision grip and Parkinson's disease. Brain 121, 1771-1784. doi: 10.1093/brain/121.9.1771

Fu, Q., Zhang, W., and Santello, M. (2010). Anticipatory planning and control of grasp positions and forces for dexterous two-digit manipulation. J. Neurosci. 30, 9117-9126. doi: 10.1523/JNEUROSCI.4159-09.2010

Gissen, A. J., Covino, B. G., and Gregus, J. (1982). Differential sensitivity of fast and slow fibers in mammalian nerve III. Effect of etidocaine and bupivacaine on fast/slow fibers. Anesth. Analg. 61, 570-575. doi: 10.1213/00000539198207000-00004

Goodman, S. R., and Latash, M. L. (2006). Feed-forward control of a redundant motor system. Biol. Cybern. 95, 271-280. doi: 10.1007/s00422-006-0086-4

Hallett, M. (1995). Is dystonia a sensory disorder? Ann. Neurol. 38, 139-140. doi: 10.1002/ana.410380203

Hartcher-O’Brien, J., Gallace, A., Krings, B., Koppen, C., and Spence, C. (2008). When vision 'extinguishes' touch in neurologically-normal people: extending the Colavita visual dominance effect. Exp. Brain Res. 186, 643-658. doi: 10. 1007/s00221-008-1272-5

Jenmalm, P., and Johansson, R. S. (1997). Visual and somatosensory information about object shape control manipulative fingertip forces. J. Neurosci. 17, 4486-4499.

Johansson, R. S., Hger, C., and Bäckström, L. (1992). Somatosensory control of precision grip during unpredictable pulling loads. III. Impairments during digital anesthesia. Exp. Brain Res. 89, 204-213. doi: 10.1007/bf002 29017
$\mathrm{BH}$ and $\mathrm{CB}$ contributed to subject screening, conducted digital nerve block procedure and assisted with data acquisition. $\mathrm{AC}$ and WZ performed data analyses, data interpretation and manuscript preparation. All authors contributed to manuscript revision, and approved the final manuscript.

\section{FUNDING}

This project was in part supported by PSC-CUNY Awards (68854-0046), and Doctoral Student Research Grant from Graduate Center of the City University of New York.

\section{ACKNOWLEDGMENTS}

We thank Bastien Schmitt and Wen Hao Wang for helpful comments on an earlier version of this write-up.

Johansson, R. S., and Westling, G. (1984). Roles of glabrous skin receptors and sensorimotor memory in automatic control of precision grip when lifting rougher or more slippery objects. Exp. Brain Res. 56, 550-564. doi: 10. 1007/bf00237997

Kaji, R., Shibasaki, H., and Kimura, J. (1995). Writer's cramp: a disorder of motor subroutine? Ann. Neurol. 38, 837-838. doi: 10.1002/ana.410380603

Kim, Y., Shim, J. K., Hong, Y. K., Lee, S. H., and Yoon, B. C. (2013). Cutaneous sensory feedback plays a critical role in agonist-antagonist co-activation. Exp. Brain Res. 229, 149-156. doi: 10.1007/s00221-013-3601-6

Koerber, H. R., and Brown, P. B. (1995). Quantitative analysis of dorsal horn cell receptive fields following limited deafferentation. J. Neurophysiol. 74, 2065-2076.

Koh, K., Kwon, H. J., Yoon, B. C., Cho, Y., Shin, J. H., Hahn, J. O., et al. (2015). The role of tactile sensation in online and offline hierarchical control of multifinger force synergy. Exp. Brain Res. 233, 2539-2548. doi: 10.1007/s00221-0154325-6

Latash, M. L., Danion, F., Scholz, J. F., and Schöner, G. (2003). "Coordination of multi-element motor systems based on motor abundance," in Progress in Motor Control: Effects of Age, Disorder and Rehabilitation (Vol. 3), eds M. L. Latash and M. F. Levin (Urbana, IL: Human Kinetics), 97-124.

Latash, M. L., Kang, N., and Patterson, D. (2002). Finger coordination in persons with Down syndrome: atypical patterns of coordination and the effects of practice. Exp. Brain Res. 146, 345-355. doi: 10.1007/s00221-002-1189-3

Latash, M. L., Li, Z. M., and Zatsiorsky, V. M. (1998). A principle of error compensation studied within a task of force production by a redundant set of fingers. Exp. Brain Res. 122, 131-138. doi: 10.1007/s002210050500

Latash, M. L., Shim, J. K., Smilga, A. V., and Zatsiorsky, V. M. (2005). A central back-coupling hypothesis on the organization of motor synergies: a physical metaphor and a neural model. Biol. Cybern. 92, 186-191. doi: 10.1007/s00422005-0548-0

Lederman, S. J., and Klatzky, R. L. (1990). Haptic classification of common objects: knowledge-driven exploration. Cogn. Psychol. 22, 421-459. doi: 10.1016/00100285(90)90009-s

Lederman, S. J., and Klatzky, R. L. (2009). Haptic perception: a tutorial. Atten. Percept. Psychophys. 71, 1439-1459. doi: 10.3758/APP.71.7.1439

Li, Z.-M., Harkness, D. A., and Goitz, R. J. (2004). Thumb force deficit after lower median nerve block. J. Neuroeng. Rehabil. 1:3. doi: 10.1186/17430003-1-3

Li, K., and Li, Z. M. (2013). Cross recurrence quantification analysis of precision grip following peripheral median nerve block. J. Neuroeng. Rehabil. 10:28. doi: 10.1186/1743-0003-10-28

Li, Z. M., and Nimbarte, A. D. (2006). Peripheral median nerve block impairs precision pinch movement. Clin. Neurophysiol. 117, 1941-1948. doi: 10.1016/j. clinph.2006.06.005

Marchese, R., Diverio, M., Zucchi, F., Lentino, C., and Abbruzzese, G. (2000). The role of sensory cues in the rehabilitation of parkinsonian patients: a comparison 
of two physical therapy protocols. Mov. Disord. 15, 879-883. doi: 10.1002/15318257(200009)15:5<879::AID-MDS1018>3.0.CO;2-9

Monzée, J., Lamarre, Y., and Smith, A. M. (2003). The effects of digital anesthesia on force control using a precision grip. J. Neurophysiol. 89, 672-683. doi: 10. 1152/jn.00434.2001

Nicolelis, M. A., Lin, R. C., Woodward, D. J., and Chapin, J. K. (1993). Dynamic and distributed properties of many-neuron ensembles in the ventral posterior medial thalamus of awake rats. Proc. Natl. Acad. Sci. U S A 90, 2212-2216. doi: 10.1073/pnas.90.6.2212

Nowak, D. A., Glasauer, S., and Hermsdörfer, J. (2003). Grip force efficiency in long-term deprivation of somatosensory feedback. Neuroreport 14, 1803-1807. doi: 10.1097/00001756-200310060-00009

Nowak, D. A., Glasauer, S., and Hermsdörfer, J. (2004). How predictive is grip force control in the complete absence of somatosensory feedback? Brain 127, 182-192. doi: 10.1093/brain/awh016

Nowak, D. A., Hermsdörfer, J., Glasauer, S., Philipp, J., Meyer, L., and Mai, N. (2001). The effects of digital anaesthesia on predictive grip force adjustments during vertical movements of a grasped object. Eur. J. Neurosci. 14, 756-762. doi: 10.1046/j.0953-816x.2001.01697.x

Pavlova, E., Hedberg, Å., Ponten, E., Gantelius, S., Valero-Cuevas, F. J., and Forssberg, H. (2015). Activity in the brain network for dynamic manipulation of unstable objects is robust to acute tactile nerve block: an fMRI study. Brain Res. 1620, 98-106. doi: 10.1016/j.brainres.2015.05.016

Quinn, L., Reilmann, R., Marder, K., and Gordon, A. M. (2001). Altered movement trajectories and force control during object transport in Huntington's disease. Mov. Disord. 16, 469-480. doi: 10.1002/mds.1108

Rasmusson, D. D. (1996). Changes in the response properties of neurons in the ventroposterior lateral thalamic nucleus of the raccoon after peripheral deafferentation. J. Neurophysiol. 75, 2441-2450. doi: 10.1002/(sici)10969861(19960101)364:1<92::aid-cne8>3.3.co;2-v

Richardson, A. G., Attiah, M. A., Berman, J. I., Chen, H. I., Liu, X., Zhang, M., et al. (2016). The effects of acute cortical somatosensory deafferentation on grip force control. Cortex 74, 1-8. doi: 10.1016/j.cortex.2015. 10.007

Rossi, S., Pasqualetti, P., Tecchio, F., Sabato, A., and Rossini, P. M. (1998). Modulation of corticospinal output to human hand muscles following deprivation of sensory feedback. Neuroimage 8, 163-175. doi: 10.1006/nimg. 1998.0352

Rossini, P. M., Rossi, S., Tecchio, F., Pasqualetti, P., Finazzi-Agrò, A., and Sabato, A. (1996). Focal brain stimulation in healthy humans: motor maps changes following partial hand sensory deprivation. Neurosci. Lett. 214, 191-195. doi: 10.1016/0304-3940(96)12940-2

Schwarz, M., Fellows, S. J., Schaffrath, C., and Noth, J. (2001). Deficits in sensorimotor control during precise hand movements in Huntington's disease. Clin. Neurophysiol. 112, 95-106. doi: 10.1016/s1388-2457(00)00497-1

Sens, E., Knorr, C., Preul, C., Meissner, W., Witte, O. W., Miltner, W. H., et al. (2013). Differences in somatosensory and motor improvement during temporary functional deafferentation in stroke patients and healthy subjects. Behav. Brain Res. 252, 110-116. doi: 10.1016/j.bbr.2013.05.048

Shim, J. K., Karol, S., Kim, Y. S., Seo, N. J., Kim, Y. H., Kim, Y., et al. (2012). Tactile feedback plays a critical role in maximum finger force production. J. Biomech. 45, 415-420. doi: 10.1016/j.jbiomech.2011.12.001

SKM, V., Zhang, W., Zatsiorsky, V. M., and Latash, M. L. (2012). Age effects on rotational hand action. Hum. Mov. Sci. 31, 502-518. doi: 10. 1016/j.humov.2011.07.005

Stone, K. D., and Gonzalez, C. L. (2014a). Grasping with the eyes of your hands: hapsis and vision modulate hand preference. Exp. Brain Res. 232, 385-393. doi: 10.1007/s00221-013-3746-3

Stone, K. D., and Gonzalez, C. L. (2014b). Grasping without sight: insights from the congenitally blind. PLoS One 9:e110175. doi: 10.1371/journal.pone. 0110175
Stone, K. D., and Gonzalez, C. L. (2015). The contributions of vision and haptics to reaching and grasping. Front. Psychol. 6:1403. doi: 10.3389/fpsyg.2015.01403

Todorov, E., and Jordan, M. I. (2002). Optimal feedback control as a theory of motor coordination. Nat. Neurosci. 5, 1226-1235. doi: 10.1038/ nn963

Touzalin-Chretien, P., Ehrler, S., and Dufour, A. (2010). Dominance of vision over proprioception on motor programming: evidence from ERP. Cereb. Cortex 20, 2007-2016. doi: 10.1093/cercor/bhp271

Wall, J. T., Kaas, J. H., Sur, M., Nelson, R. J., Felleman, D. J., and Merzenich, M. M. (1986). Functional reorganization in somatosensory cortical areas $3 \mathrm{~b}$ and 1 of adult monkeys after median nerve repair: possible relationships to sensory recovery in humans. J. Neurosci. 6, 218-233.

Weiss, T., Sens, E., Teschner, U., Meissner, W., Preul, C., Witte, O. W., et al. (2011). Deafferentation of the affected arm a method to improve rehabilitation? Stroke 42, 1363-1370. doi: 10.1161/STROKEAHA.110.601138

Witney, A. G., Wing, A., Thonnard, J. L., and Smith, A. M. (2004). The cutaneous contribution to adaptive precision grip. Trends Neurosci. 27, 637-643. doi: 10. 1016/j.tins.2004.08.006

Zatsiorsky, V. M., Latash, M. L., Gao, F., and Shim, J. K. (2004). The principle of superposition in human prehension. Robotica 22, 231-234. doi: 10 1017/S0263574703005344

Zhang, W., Gordon, A. M., Fu, Q., and Santello, M. (2010). Manipulation after object rotation reveals independent sensorimotor memory representations of digit positions and forces. J. Neurophysiol. 103, 2953-2964. doi: 10.1152/jn. 00140.2010

Zhang, W., Johnston, J. A., Ross, M. A., Coakley, B. J., Gleason, E. A., Dueck, A. C., et al. (2012). Effects of carpal tunnel syndrome on adaptation of multi-digit forces to object mass distribution for whole-hand manipulation. J. Neuroeng. Rehabil. 9:83. doi: 10.1186/1743-0003-9-83

Zhang, W., Johnston, J. A., Ross, M. A., Sanniec, K., Gleason, E. A., Dueck, A. C., et al. (2013). Effects of carpal tunnel syndrome on dexterous manipulation are grip type-dependent. PLoS One 8:e53751. doi: 10.1371/journal.pone. 0053751

Zhang, W., Johnston, J. A., Ross, M. A., Smith, A. A., Coakley, B. J., Gleason, E. A., et al. (2011). Effects of carpal tunnel syndrome on adaptation of multidigit forces to object weight for whole-hand manipulation. PLoS One 6:e27715. doi: 10.1371/journal.pone.0027715

Zhang, W., Olafsdottir, H. B., Zatsiorsky, V. M., and Latash, M. L. (2009). Mechanical analysis and hierarchies of multi-digit synergies during accurate object rotation. Motor Control 13, 251-279. doi: 10.1123/mcj.13.3.251

Zhang, W., and Santello, M. (2014). "Quantification of behavioral consequences of carpal tunnel syndrome: insights from biomechanical analysis of grasping and manipulation," in Carpal Tunnel Syndrome: Risk Factors, Symptoms and Treatment Options, ed. M. Ledford (New York, NY: Nova Science Publishers), 33-55.

Zhang, W., Scholz, J. P., Zatsiorsky, V. M., and Latash, M. L. (2008). What do synergies do? Effects of secondary constraints on multidigit synergies in accurate force-production tasks. J. Neurophysiol. 99, 500-513. doi: 10.1152/jn. 01029.2007

Conflict of Interest Statement: The authors declare that the research was conducted in the absence of any commercial or financial relationships that could be construed as a potential conflict of interest.

Copyright $\odot 2016$ Carteron, McPartlan, Gioeli, Reid, Turturro, Hahn, Benson and Zhang. This is an open-access article distributed under the terms of the Creative Commons Attribution License (CC BY). The use, distribution and reproduction in other forums is permitted, provided the original author(s) or licensor are credited and that the original publication in this journal is cited, in accordance with accepted academic practice. No use, distribution or reproduction is permitted which does not comply with these terms. 